\title{
A CATALOGUE OF THE DIVING BEETLES (COLEOPTERA: ADEPHAGA, DYTISCIDAE) OF THE REPUBLIC OF MOLDOVA
}

\author{
Neculiseanu Zaharia \\ Institute of Zoology of MECC, Chisinau, Republic of Moldova \\ e-mail:zneculiseanu@yahoo.com \\ https://doi.org/10.53937/9789975315975.56
}

The family DYTISCIDAE is worldwide in distribution. The species are common in tropical latitudes and increasingly common toward the temperate zone. The latest and updated world catalog of the Dytiscidae family is presented by Nilsson (2015). Today more than 160 genera are known globally encompassing more than 4000 species.

This paper presents the first complete and updated list of diving beetles (Coleoptera, Dytiscidae) found and published in Republic of Moldova until 2018. Catalogue includes 7 subfamilies, 11 tribes, 16 genera and 29 species. The species marked with asterisk are new for the fauna of the Republic of Moldova.

Family DYTISCIDAE Leach, 1815

Subfamily AGABINAEThomson, 1867

Tribe AGABINIThomson, 1867

Genus Agabus Leach, 1817 - 2 species

Subgenus Agabus Leach, 1817

uliginosus (Linnaeus, 1761)

Subgenus Gaurodytes Thomson, 1859

Guttatus (Paykull, 1798)

Genus Ilybius Erichson, 1832 - 3 species

Ater (De Geer, 1774)*

guttiger (Gyllenhal, 1808) *

subaeneus Erichson, 1837 
Subfamily COLYMBETINAE Erichson, 1837

Tribe COLYMBETINI Erichson, 1837

Genus Colymbetes Clairville, 1806 - 2 species

fuscus (Linnaeus, 1758)

striatus LeConte, 1852*

Genus Rhantus Dejean, 1833 - 2 species

exsoletus (Forster, 1771)

suturalis (W.S. Macleay, 1825)

Subfamily COPELATINAE Van den Branden, 1885

Tribe COPELATINI Van den Branden, 1885

Genus Liopterus Dejean, 1833 - 1 species

haemorrhoidalis (Fabricius, 1787)

Subfamily CYBISTRINAE Sharp, 1880

Tribe CYBISTRINI Sharp, 1880

Genus Cybister Curtis, 1827 - 1 species

Subgenus Cybister Curtis, 1827

lateralimarginalis (De Geer, 1774)

Subfamily DYTISCINAE Leach, 1815

Tribe ACILIINI Thomson, 1867

Genus Acilius Leach, 1817 - 1 species

Subgenus Acilius Leach, 1817

sulcatus (Linnaeus, 1758)

Genus Graphoderus Dejean, 1833 - 1 species

cinereus (Linnaeus, 1758)

Tribe DYTISCINI Leach, 1815

Genus Dytiscus Linnaeus, 1758 - 4 species

Circumcinctus Ahrens, 1811

circumflexus Fabricius, 1801

dimidiatus Bergsträsser, 1778

marginalis Linnaeus, 1758 
Tribe HYDATICINI Sharp, 1880

Genus Hydaticus Leach, 1817 - 1 species transversalis (Pontoppidan, 1763)

Subfamily HYDROPORINAE Aube, 1836

Tribe BIDESSINI Sharp, 1880

Genus Hydroglyphus Motschulsky, 1853 - 1 species

Geminus (Fabricius, 1792)

Tribe HYDROPORINI Aube, 1836

Subtribe HYDROPORINA Aube, 1836

Genus Hydroporus Clairville, 1806 - 3 species

angustatus Sturm, 1835

fuscipennis Schaum, 1868

obscurus Sturm, 1835

Subtribe SIETTITIINA Smrž, 1982.

Genus Graptodytes Seidlitz, 1887 - 1 species

Bilineatus (Sturm, 1835)

Genus Porhydrus Guignot, 1945 - 1 species

lineatus (Fabricius, 1775)

Tribe HYGROTINI Portevin, 1929

Genus Hygrotus Stephens, 1828 - 3 species

Subgenus Coelambus Thomson, 1860

confluens (Fabricius, 1787)

impressopunctatus (Schaller, 1783)

parallellogrammus (Ahrens, 1812)

Subfamily LACCOPHILINAE Gistel, 1848

Tribe LACCOPHILINI Gistel, 1848

Genus Laccophilus Leach, 1815 - 2 species

minutes (Linnaeus, 1758)

poecilus Klug, 1834

Studiile au fost realizate în cadrul proiectului instituțional fundamental 15.817.02.12F. 